\title{
Ultrastructure of mitochondrial nucleoid and its surroundings
}

\author{
Jarmil Prachař \\ Laboratory of Tumor Immunology, Cancer Research Institute, Slovak Academy of Sciences, Vlárska 7, 83391 Bratislava, \\ Slovak Republic
}

\begin{abstract}
Mitochondrial nucleoids (hereafter nucleoids) contain genetic information, mitochondrial DNA, prerequisite for mitochondrial functioning, particularly information required for mitochondrial electron transport. To understand nucleoid functioning, it is imperative to know its ultrastructure and dynamics in the context of the actual mitochondrial state. In this study, we document the internal structure, different positions of nucleoids inside the mitochondrial tube and their different morphology. The nucleoid cores appear in section as circular or slightly oval objects ranging from 50 to $100 \mathrm{~nm}$ in diameter. They are mainly located in the matrix between cristae inside the mitochondrial tube but they are also frequently found close to the inner mitochondrial surface. In tightly packed form, their interior exhibits sophisticated nucleoprotein regularity. The core surroundings form an electron-lucent thick layer which is probably partitioned into separate chambers. We suggest that the morphology of nucleoids mirrors the mode of energy production, glycolysis versus oxidative phosphorylation. The new high resolution transmission electron microscopy method enabled us to obtain morphological characteristics on yet unpublished level.
\end{abstract}

Key words: Mitochondrial nucleoid - Electron-dense core - Electron-lucent envelope - High resolution transmission electron microscopy

Abbreviations: mtDNA, mitochondrial DNA; TFAM, mitochondrial transcription factor-A.

\section{Introduction}

Nucleoids are supramolecular objects providing mitochondrial maintenance and function. They contain one or more molecules of mitochondrial DNA (mtDNA) and this makes them usually highly electron-dense, as observed also in other objects with high nucleic acid content. They bind heavy metal ions, which improves contrast, similarly as in heterochromatin, chromosome, nucleolus, and perichromatin granules. While many nucleoids are so tightly packed that they show even higher electron-density than heterochromatin, in highly active mitochondria, many are hardly recognizable in the granular matrix (Prachař 2010).

In addition to DNA, nucleoids contain a large number of diverse proteins. Some of them are abundant, while others

Correspondence to: Jarmil Prachař, Laboratory of Tumor Immunology, Cancer Research Institute, Slovak Academy of Sciences, Vlárska 7, 83391 Bratislava, Slovak Republic

E-mail: jarmil.prachar@savba.sk are barely detectable (reviewed in Holt et al. 2007; Bogenhagen et al. 2008; Bogenhagen 2012; Kukat and Larsson 2013; Wessels et al. 2013; Hensen et al. 2014).

Recent studies established nucleoids as control centers for mitochondrial biogenesis. Many fundamental processes essential for mitochondrial maintenance and function are performed in the vicinity of the nucleoid. This concerns executive proteins involved in replication, repair, transcription, RNA post-transcriptional modifications and cleavage, which have been shown to be physically bound, directly or indirectly, to the nucleoid. In addition, the first steps in the assembly of mitochondrial ribosome subunits are also performed near the nucleoid (Lee et al. 2013; Bogenhagen et al. 2014).

The nucleoid contains one or more mtDNA copies, with estimates of up to 10 copies per nucleoid (reviewed in Spelbrink 2010). Using high resolution STED microscopy (Stimulated Emission Depletion Microscopy, recently developed superresolution technique), Kukat et al. (2011) illustrated that nucleoids were often present in clusters, previously considered 
a single nucleoid. This group concluded that the number of mtDNA copies per nucleoid is mostly only one. However, since nucleoids differ in size, it can be assumed that the mtDNA copy number also differs, with an average that is per nucleoid lower than previously assessed by other authors.

The number of nucleoids in in vitro cultivated cells was estimated to be in the range of 450-800 per cell (Iborra et al. 2004; Legros et al. 2004), but high resolution STED microscopy revealed an approximate average of 1.6 times more nucleoids per cell (Kukat et al. 2011). Different reports recorded the nucleoid size to range from $50 \mathrm{~nm}$ to $300 \mathrm{~nm}$ (Brown et al. 2011; Kukat et al. 2011; Bogenhagen 2012; Kopek et al. 2012; Tauber et al. 2013). This wide range can be attributed to their actual diversity as well as to different methodological approaches employed, and most probably both factors may be involved.

We have used new technical approaches which enable to observe objects in cell section at very high resolution, about three times higher than ever published. These new protocols of sample preparation and microscope handling allow to obtain a detailed view of the nucleoid interior and its surroundings.

We observed highly packed nucleoids in the L1210 leukemia cell line, which uses glycolysis as an energy source. We showed for the first time that these nucleoids are composed from electron-dense core surrounded by an electron-lucent thick layer, most likely partitioned into separate chambers.

While some nucleoprotein packing regularity was noted in the electron-dense nucleoids in the leukemia cell line L1210, the nucleoids in highly protein-synthetically active Caco-2 colon cancer cells were less electron-dense, with diffuse edges, and without any sign of regularity. As a major energy source, different cancer cells can use both glycolysis and oxidative phosphorylation (reviewed in Ježek et al. 2010). Here we suggest that different nucleoid morphology may reflect different modes or intensity of energy metabolism.

\section{Materials and Methods}

\section{Cell cultures}

The mouse leukemia cell line L1210 (purchased from the American Type Culture Collection, Rockville, MD, USA) was maintained in RPMI 1640 medium supplemented with $10 \%$ fetal bovine serum. The cells were fixed at $10^{6} / \mathrm{ml}$ cell density while still dividing, yet after the exponential growth phase.

The human colon adenocarcinoma cell line Caco-2 was obtained from the European Collection of Cell Culture (Wiltshire, UK). The cells were cultured in RPMI 1640 medium supplemented with $10 \%$ fetal bovine serum and collected for electron microscopy study in the early exponential growth phase. Since these cells adhere very strongly to plastic, glass dishes were used for cultivation to prevent loss of the bottom cellular membrane. The cells were fixed without washing with $3 \%$ glutaraldehyde in PBS for 1 hour and then scraped following osmium tetroxide postfixing. Caco-2 cells have already been described in detail (Prachar 2010).

In this study, the same experiments were used as described previously (Prachar 2010), yet newly developed protocols were applied for sample preparation, for handling the microscope, and for image recording.

\section{Preparation of samples for transmission electron microscopy}

The L1210 cells ( $5 \mathrm{ml}$ ) were quickly pelletized ( $2 \mathrm{~min}, 100 \mathrm{~g}$ ) and immediately fixed without washing. The Caco-2 cells were treated in the same way, but after the postfixing step (see above). Fixation, contrasting, embedding and staining of sections were performed conventionally. Briefly the procedure was as follows: fixation with $3 \%$ glutaraldehyde in PBS for $1 \mathrm{~h}$, postfixation in $1 \% \mathrm{OsO}_{4}$ for $1 \mathrm{~h}$, washing 3 times with cold deionized water and contrasting with a saturated solution of uranyl acetate in deionized water overnight. For washing cells were resuspended in water and shortly centrifuged 3 times between each step. Subsequently, the cells were resuspended in low melting point agarose ( $1 \%$ in water) at $37^{\circ} \mathrm{C}$, pelletized and kept in an ice bath for a short time, as required for embedment in agarose. The following step was dehydration in $70 \%$ and $100 \%$ ethyl alcohol and equilibration in propylene oxide, with subsequent embedding in Poly/Bed 812 epoxy resin (Polysciences). For preparation of the epoxy resin, a combination of components was chosen to achieve maximal hardness, according to the manufacturer's instructions. The sample in epoxy resin was kept at $37^{\circ} \mathrm{C}$ for $48 \mathrm{~h}$ to harden, and then at $50^{\circ} \mathrm{C}$ for the next $24 \mathrm{~h}$. Ultrathin sections were picked up directly on bare 700 or 1000 mesh copper grids (Agar Scientific). Section thickness was approximately $50 \mathrm{~nm}$ or less. Contrasting of the ultrathin sections (one side only) was done on a drop $(50 \mu \mathrm{l})$ of a saturated solution of uranyl acetate in water at $37^{\circ} \mathrm{C}(2 \mathrm{~h})$ and, after intensive washing in deionized water, the last step was contrasting on a drop of Reynolds solution at laboratory temperature (4 min or less). Unless otherwise stated, all procedures were carried out at room temperature. Uranyl acetate and Reynolds solution were prepared the same day as contrasting was done.

Images were collected using JEOL JEM-1200 EX transmission electron microscope at $80 \mathrm{kV}$ with Kodak electron microscope films 4489 .

\section{Handling of the microscope to attain high resolution in cell sections}

Our high resolution method is based on two microscope settings. The first is the setting most commonly used by 
biologists. The second setting is used for recording, and this favors high resolution without defects from radiation damage. This high resolution methodology was recently published in detail (Prachar 2014) and it is also discussed in the "Discussion" section.

\section{Film grain}

We enlarged negative films in a photographic enlarger approximately 2.3 times. The positive photographs were scanned and relevant parts of the micrograph were further enlarged in the computer approximately 10 times, thus providing a total original negative film enlargement of up to 25 times. We decided to determine what noise can be caused by the film grain and thus observed a processed Kodak EM 4489 film under the light microscope. Granularity appears first as a very fine texture at $40 \times$ magnification. Thus, consistent with our results, the film grain caused no noise. It was quite surprising that we observed no film noise even in much higher enlargements. This was presumably due to an "overwriting" of the very light texture by the dark, overexposed negative image.

\section{Results}

Morphological definition of the nucleoid based on transmission electron microscopy

We suggest to define the mitochondrial nucleoid according to the recorded electron density and in case of packed nucleoids in L1210 we have to consider also electron-lucent surroundings which wraps the nucleoid core. Hereafter, we will therefore consider the electron-dense core, which contains mtDNA plus electron-lucent layer around it, nucleoid. We describe both parts of the nucleoid separately.

Nucleoid cores appear as electron-dense circular or oval objects of approximately $50 \mathrm{~nm}$ to maximum $100 \mathrm{~nm}$ in diameter (Figs. 1-8). The electron density is caused by the presence of a large number of negatively charged DNA phosphate groups which bind heavy metal cations, especially uranium (see "Materials and Methods" section). The diameter size depends on where the core is cut. If the section runs off-center, a false smaller value can be recorded, while the real diameter is actually larger. Some smaller values may thus represent spherical caps of the nucleoid core bodies caused by sectioning off-center. However, from the experience with sections of viral particles of similar diameter as cores, this error appears only in a small minority of particles. Therefore, the majority of nucleoid cores possess a real diameter smaller than $100 \mathrm{~nm}$. This is in agreement with the recently published estimation of nucleoid mean size $70 \mathrm{~nm}$ (Kukat et al. 2011).
In L1210 cells, the core is located approximately centrally in the larger electron-lucent body, which creates the electronlucent layer around the core. The electron-lucent layer or envelope is well recognizable in most but not all nucleoids, probably depending on the activity of the nucleoid. The situation is different in Caco-2 cells (see below).

The diameter of the whole nucleoid body, core plus electron-lucent layer, is the sum of the core diameter plus thickness of the layer. Because different nucleoid cores have different diameters, the diameters of the entire bodies are also different. For example, in Figure 1A, the core has diameter about $87 \mathrm{~nm}$ and the whole nucleoid diameter is $130 \mathrm{~nm}$. In Figure 8A, the length of the core along longer axis of the oval is $114 \mathrm{~nm}$ and the length of the whole nucleoid in the same place is about $160 \mathrm{~nm}$. The thickness of the layer in the nucleoid presented in Figure 4 averages $18 \mathrm{~nm}$ and the diameter of the nucleoid core is $50 \mathrm{~nm}$ then the diameter of the whole nucleoid is about $86 \mathrm{~nm}$. The thickness of the layer exhibit considerable variability (see for example Fig. $8 \mathrm{~A})$. The electron-lucent layer was referred to as a "halo" in our previous work (Prachař 2010).

\section{DNA occurrence in the nucleoid core}

To obtain a direct proof of DNA by transmission electron microscopy alone presents a problem. The high electron density of the objects containing nucleic acids is in accordance with our finding but it is still no direct proof. The best proof would be monitoring of mitochondrial DNA depletion (SeidelRogol and Shadel 2002) by light microscopy (e.g. confocal) and electron microscopy in parallel. However, this approach is currently beyond our possibilities. Immuno-electron microscopy would be a suitable method to localize nucleoids but this method uses very mild contrasting procedures and is not ideal for identification of the object. The images of nucleoids would be very different from those obtained by standard contrasting methods (see Iborra et al. 2004). Anyway, immuno-EM is also beyond our possibilities.

Nevertheless, we developed the methodology of extremely high resolution, which allows to visualize the section surface at the molecular level (Figs. 1B, 8C,D). First we saw an illegible pattern of various patches. Later on, we compared the sections through nucleoids, heterochromatin, chromosomes, nucleolus, perichromatin granules and ribosomes. All these sections show common features. Enlargements of high resolution micrographs show basically three shades of patches - white (or light), gray and dark (or black). The brightness depends on the photographic procedure, but the substance remains the same. Comparison of an enlarged section through the nucleoid depicted in Fig. 1A at lower enlargement and the section through heterochromatin is presented in Fig. 1B. Since the proteins are electron-lucent and the deposits of heavy metals are 
dark, the gray patches should represent phosphate groups of nucleic acids. We therefore concluded that the nucleoids studied were composed of proteins and nucleic acid(s). We can, however, not exclude that the nucleic acid present is RNA since ribosomes display a similar gray-shade pattern (not shown). We assume that we observed nucleoids, not only on the basis of our high resolution images, but also in light of the vast majority of identical features found in different laboratories by different methods, as well as by considering the staining typical for nucleoproteins using lower resolution.

\section{Nucleoid position in the mitochondrial tube}

Model systems used, both here and in our previous work, located nucleoids most frequently among the cristae inside the mitochondrial tube (Figs. 1A, 4, 5 and 7; Prachař 2010). Approximately $10 \%$ of the nucleoids in L1210 cells were close to the inner mitochondrial membrane near the surface (Figs. 6 and 8A; 94 nucleoids evaluated; see also Prachar 2010). In light of these findings, we can consider a random location of nucleoids in the mitochondrial matrix in a plane perpendicular to the mitochondrial tube axis. The position
A

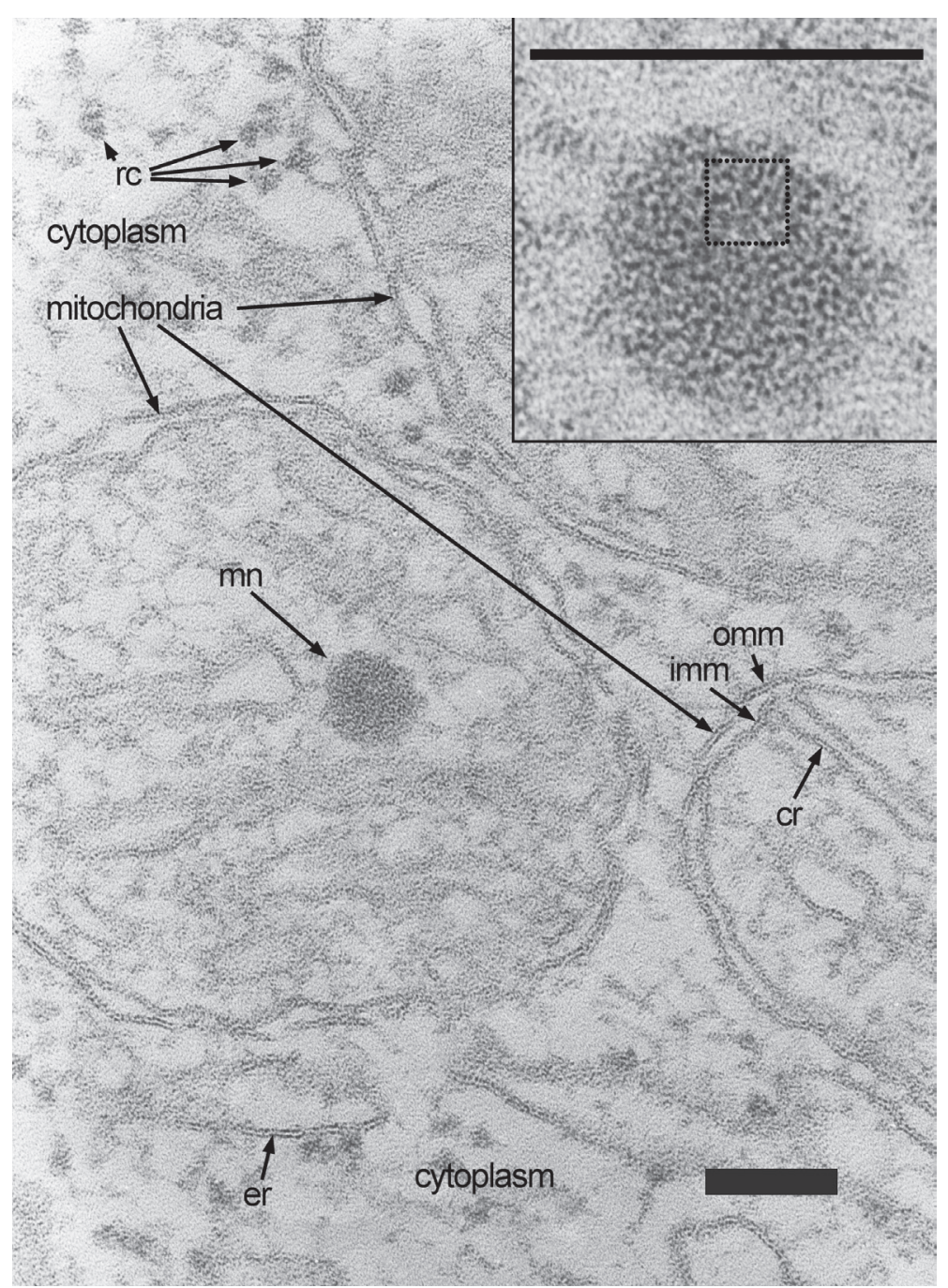

B

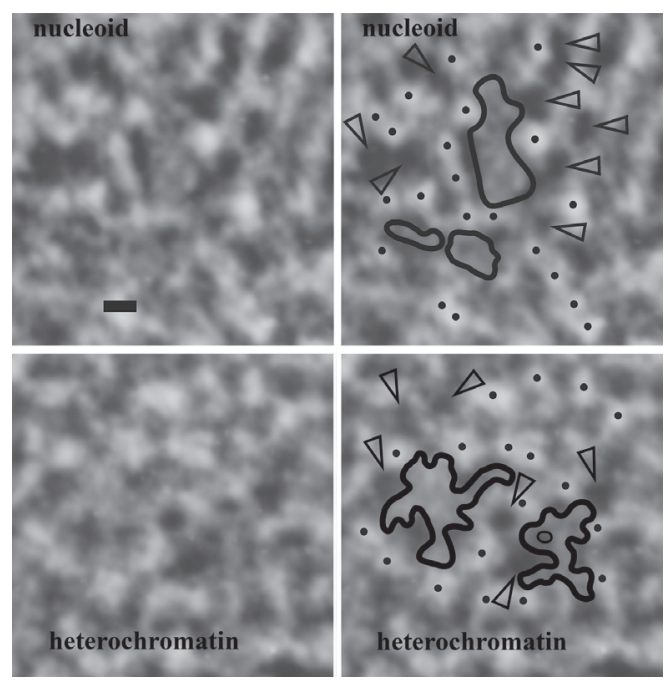

Figure 1. A. L1210 cell section showing parts of three mitochondria. The packed nucleoid is located between cristae in one of them. Higher enlargement is shown in the inset and the dotted rectangle denotes the area which is further enlarged in Fig. 1B. Original magnification: $\times 75,000$; scale bars: $100 \mathrm{~nm}$. $\mathrm{mn}$, nucleoid (nucleoid core in lighter surroundings); omm, outer mitochondrial membrane; imm, inner mitochondrial membrane; cr, crista; er, endoplasmic reticulum; rc, cytoplasmic ribosomes. B. Comparison of the section through the nucleoid presented in (A) and the section through the heterochromatin. The upper part shows the enlarged part of the nucleoid section presented in (A). Three shades of patches - white (or light), gray and dark (or black) are depicted by dots, contours and by arrowheads, respectively, on the right part of the figure (for clarity, only some patches are depicted). Contours show presumable nucleic acid(s), the dots indicate presumed proteins and the dark patches are contrast agent deposits. The lower part of the picture depicts a section through L1210 cell heterochromatin from the same grid. The section through heterochromatin is lighter and has lower contrast but the shades of gray show a similar pattern as in the case of nucleoid. Scale bar: $2 \mathrm{~nm}$. 
of the nucleoids in the direction longitudinally to the tube axis cannot be determined by our method because the cell sections used in electron microscopy are too thin.

\section{Nucleoid anchored in membranes}

Of 124 nucleoids evaluated in L1210 cells only one was anchored in crista membranes (Fig. 2). This is most likely an underestimation because of the low probability of sectioning through the narrow anchor. Although we are fully aware of this type of anchorage, we can only speculate on a raison d'être.

Anchorage in surface mitochondrial membranes is depicted in Figure 3. The bulb-like nucleoid possesses an an-

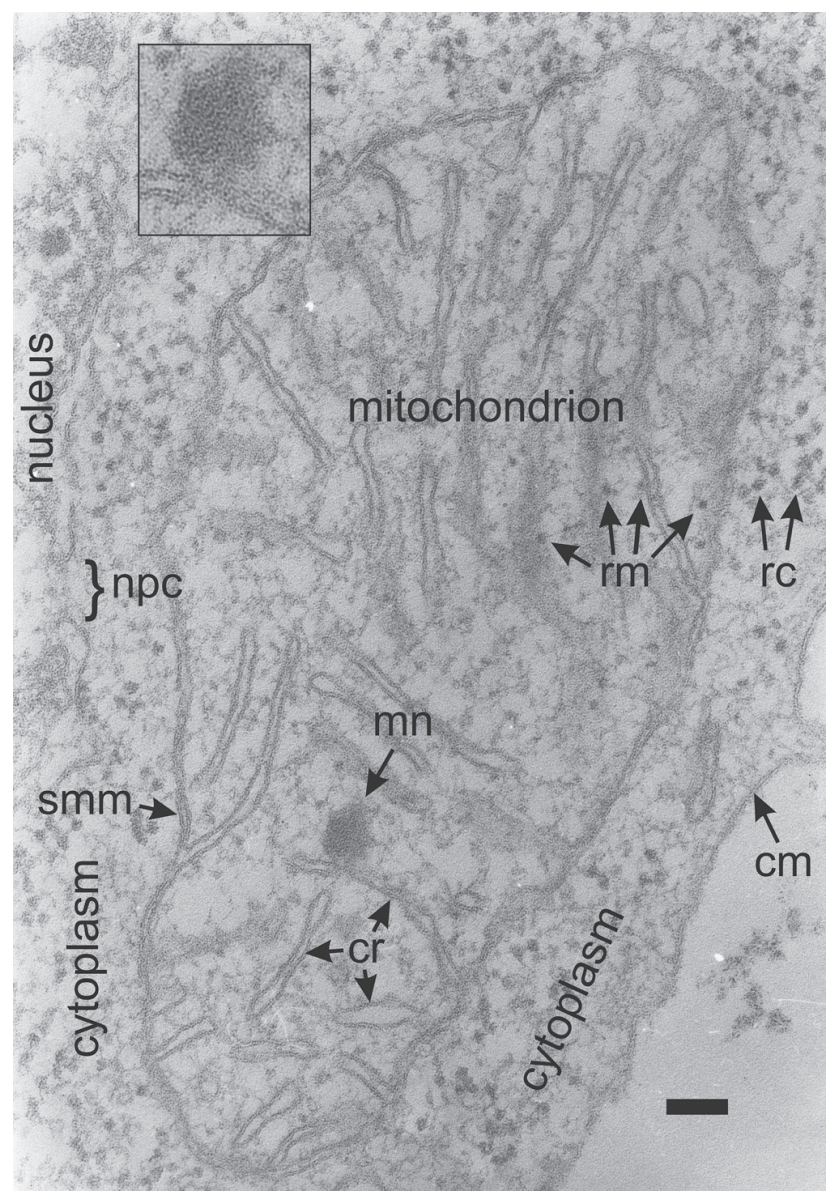

Figure 2. Section through an L1210 cell mitochondrion showing a nucleoid anchored in the crista membrane. The inset gives twofold nucleoid enlargement. Original magnification: $\times 40,000$; scale bar: $100 \mathrm{~nm} . \mathrm{mn}$, nucleoid (only core; the lighter surrounding is not distinguishable); cr, cristae; $\mathrm{rm}$, mitochondrial ribosomes; smm, surface mitochondrial membranes (outer and inner boundary membranes); rc, cytoplasmic ribosomes; $\mathrm{cm}$, cytoplasmic membrane; npc, nuclear pore complex. chor of lower electron density going through both the inner and outer mitochondrial membrane. Of the 124 nucleoids evaluated, we found four anchored in surface membranes, two of which were shown in our earlier work (Prachař 2010). This frequency of occurrence is also underestimated because of the low probability of sectioning through the narrow anchor. A further reduction in frequency may be due to the fact that the cell culture in our model was after the exponential phase (see "Discussion" section).

\section{Electron-lucent layer surrounding the nucleoid core}

The light layer surrounding the nucleoid core is almost always discernible in L1210 cells. The L1210 cell mitochondrial matrix is light and it is clearly apparent that the light layer around the nucleoid cores is in contact with membranes of cristae. The light layer has also a certain border between cristae composed of more electron-dense matter (Fig. 4). Faint "spokes" in the layer can be distinguished. Their

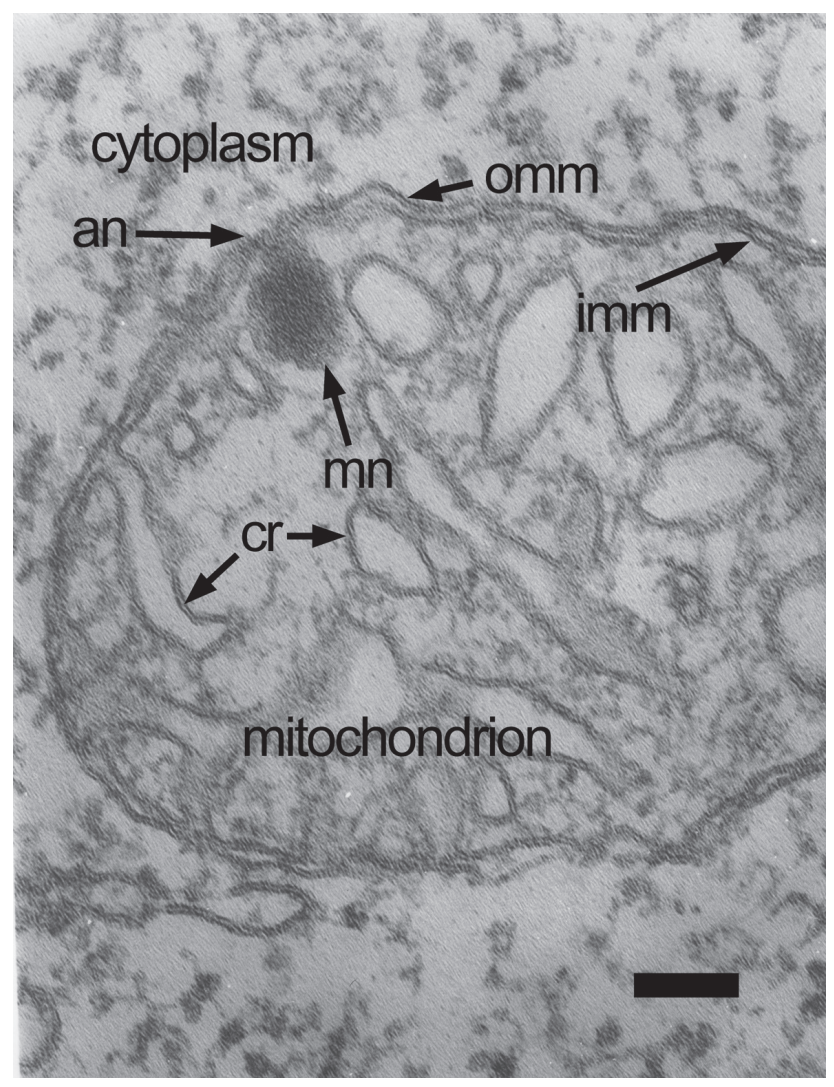

Figure 3. Section through an L1210 cell mitochondrion showing bulb-shaped nucleoid anchored in both inner and outer mitochondrial membranes. Original magnification: $\times 75,000$; scale bar: $100 \mathrm{~nm}$. mn, nucleoid (nucleoid core on electron-lucent background); omm, outer mitochondrial membrane; imm, inner mitochondrial membrane; $\mathrm{cr}$, crista; an, anchor. Note: the image has conventional resolution. 
fuzziness indicates that they are most likely of protein rather than membrane origin. Although they have a small width, they are mostly visible without interruption connecting the nucleoid and crista membrane. Therefore in $3 \mathrm{D}$ projection, the "spokes" are boundary walls between the vesicles or small chambers rather than being sticks only. The vesicular nature of the nucleoid core surroundings was even more clearly visible in our previous work, where the nucleoid with chambers resembles a sunflower (see Fig. 5 in Prachař 2010).

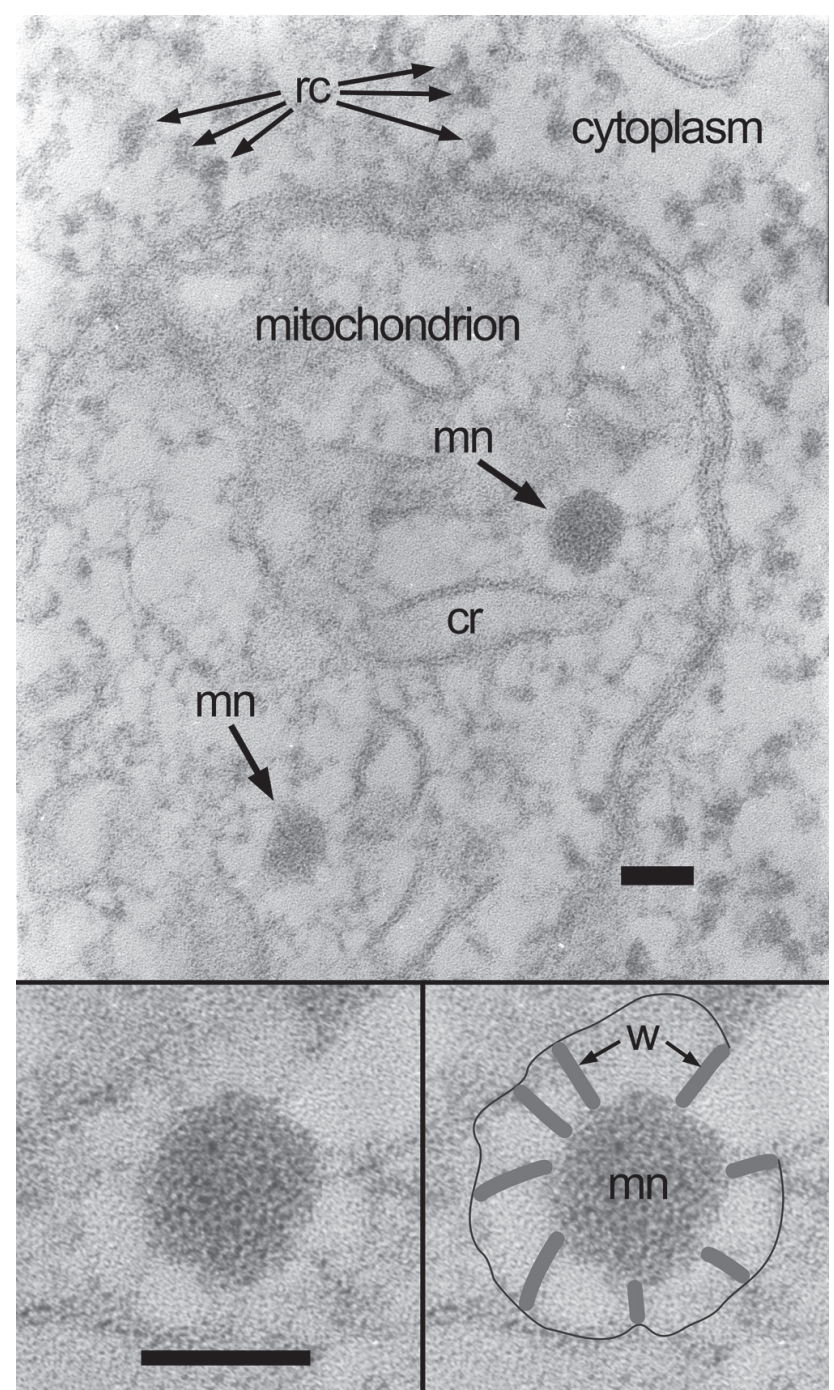

Figure 4. Section of an L1210 cell showing a mitochondrion with two sectioned nucleoids. The packed nucleoids are located between cristae and their cores are surrounded by an electron-lucent thick layer. The enlarged upper nucleoid is in the lower part of the image. The presumed chamber walls and layer border are outlined on the right side. Original magnification: $\times 100,000$; scale bars: $50 \mathrm{~nm}$. $\mathrm{mn}$, nucleoid (nucleoid core covered with electron-lucent envelope); cr, crista; rc, cytoplasmic ribosomes; $\mathrm{w}$, presumed walls between the chambers in the light body.
We estimate the total number of chambers surrounding one nucleoid core to be in tens of units.

In contrast to L1210 cells, Caco-2 cells are highly active in proteosynthesis and cultured cells produce mucus into the medium exactly as they did in the human colon. Their mitochondrial matrix is granulous and considerably electron-dense, but the nucleoid cores are less electron-dense than in L1210 cells and they never reach $100 \mathrm{~nm}$ in diameter (Fig. 5). Lower electron density indicates lower DNA content. The edges of these nucleoids are diffuse in comparison with those in L1210 cells. The light layer analogous to L1210 cells are mostly unrecognizable. Some nucleoid cores are surrounded by a recognizable lighter zone, but this region contains granulous material. These situations might represent actively transcribed nucleoids. Some nucleoids may also

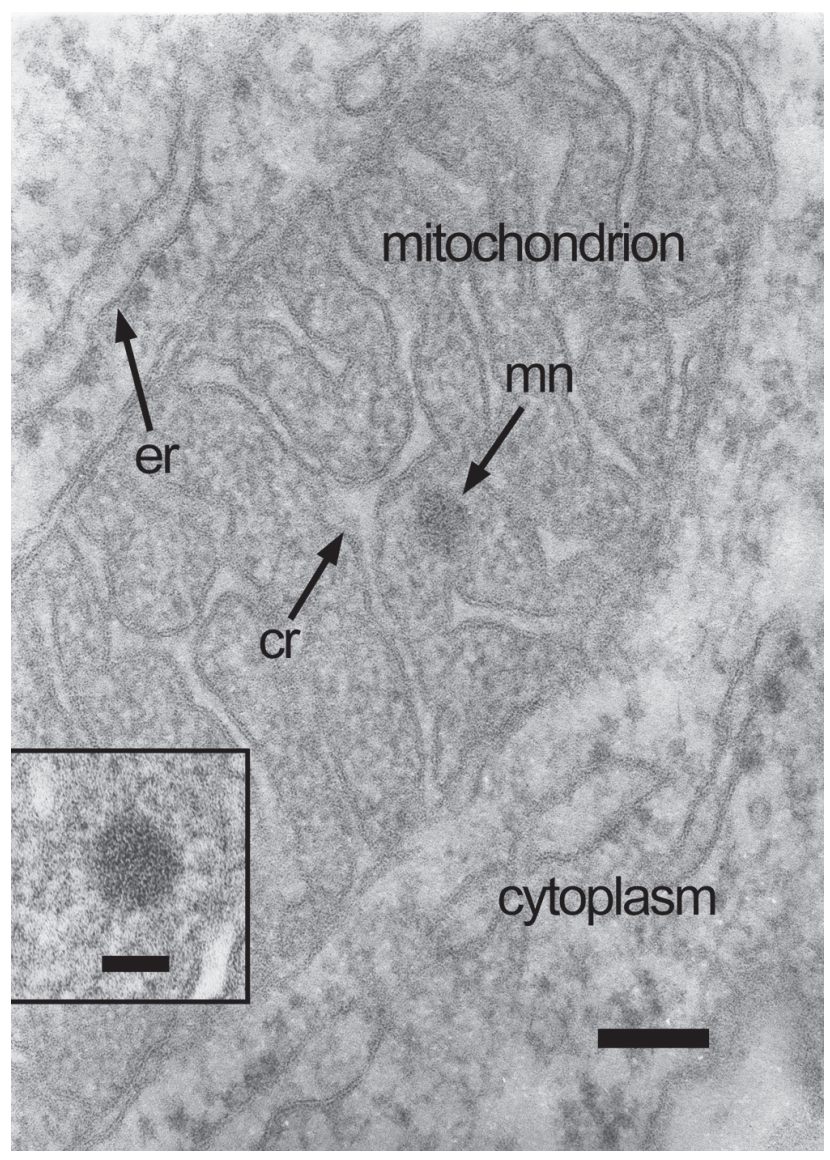

Figure 5. Section of Caco-2 cell showing mitochondrion with a nucleoid with diffuse edges located in granulous matrix between cristae. A nucleoid core from a different mitochondrion is shown in the inset, where the close granulous surrounding has a lighter background and is distinguishable from the matrix. Original magnification: $\times 75,000$; scale bar: $100 \mathrm{~nm}$; scale bar in the inset: $50 \mathrm{~nm}$. mn, nucleoid (only core without electron-lucent surroundings); cr, crista; er, endoplasmic reticulum. Note: the image has conventional resolution. 
be anchored in the surface membranes, but this is not easily recognizable because matrix granulous material is present (Prachař 2010).

\section{Efforts to find regularity in the arrangement of nucleoid nucleoprotein}

Nucleoid core often exhibit higher electron density than heterochromatin in L1210 cells at the end of the exponential phase or than chromosomes in anaphase. This may explain our endeavor to investigate the ultrastructural organization of the nucleoid in great detail, but we discerned unclear signs of regular arrangement on the molecular complex level only once in over 100 inspected nucleoids (Fig. 6).

We first used common microscope settings which, however, do not favor observations at the molecular level, and the use of higher magnification above $100,000 \times$ caused radiation damage, as seen in Figures 6 and 7. Very small structures merge and some disappear completely. The image ceases to be authentic especially concerning the contrast of different structures.

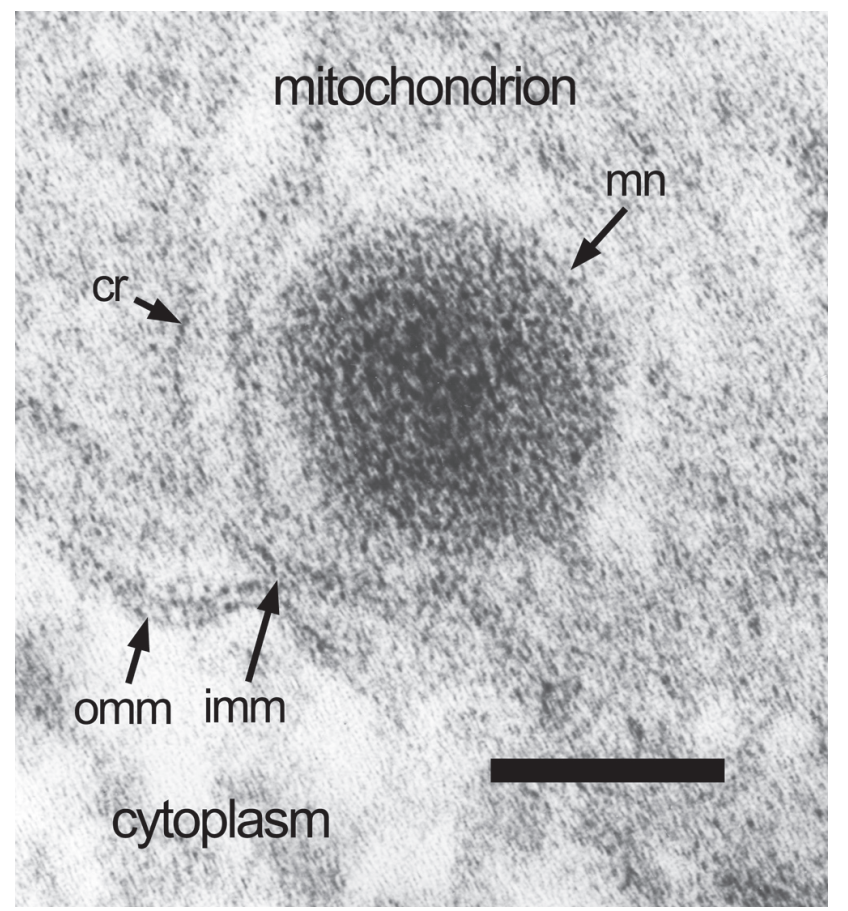

Figure 6. Section of an L1210 cell showing part of the mitochondrion with a packed nucleoid near the inner mitochondrial surface. The nucleoid shows certain signs of regular arrangement. Original magnification: $\times 250,000$; scale bar: $50 \mathrm{~nm}$. mn, nucleoid (nucleoid core on lighter background); omm, outer mitochondrial membrane; imm, inner mitochondrial membrane; cr, crista. Note: the image has conventional resolution and shows considerable radiation damage.
We found many heavily compacted nucleoids in serumstarved cells (Prachař 2010), so we assumed that DNA held together with proteins may show some regular organization. However, we found only one instance of certain concentric regularity in the 100 nucleoids inspected (Fig. 7).

We then developed our high resolution method to detail structures at molecular level without causing radiation damage (see "Material and Methods"). Using this method, of the 30 examined nucleoids in serum-nourished L1210 cells at the end of exponential phase, we found one nucleoid close to the mitochondrial inner surface with very regular organization of molecular complexes (Fig. 8A). The nucleoid core shows six rows of white and gray spots bent on both the top and bottom parts of its body (Fig. 8B). This possessed the regular and sophisticated nucleoprotein coiling. The midlines of each row were approximately $6 \mathrm{~nm}$ apart, but this varied considerably. Although the picture appears to be three-dimensional and

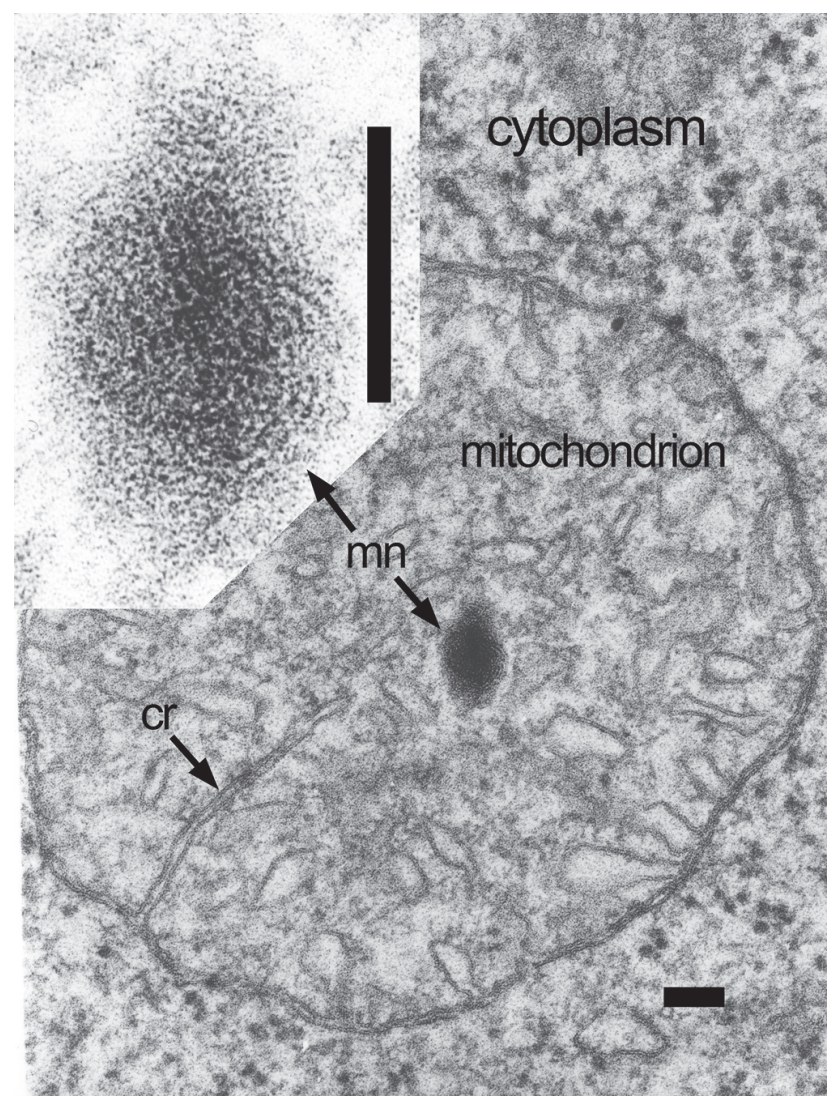

Figure 7. Section of an L1210 cell showing a packed nucleoid located between cristae. The cell was serum-starved for 24 hours (Prachar 2010). Higher enlargement in the inset shows concentric filament arrangement; this record was enlarged from different micrograph of the same identical area taken with original magnification of 150,000x. Original magnification of the micrograph with whole mitochondrion: $\times 75,000$; scale bars: $100 \mathrm{~nm}$. mn, nucleoid (nucleoid core on lighter background); cr, crista. Note: the image has conventional resolution. 

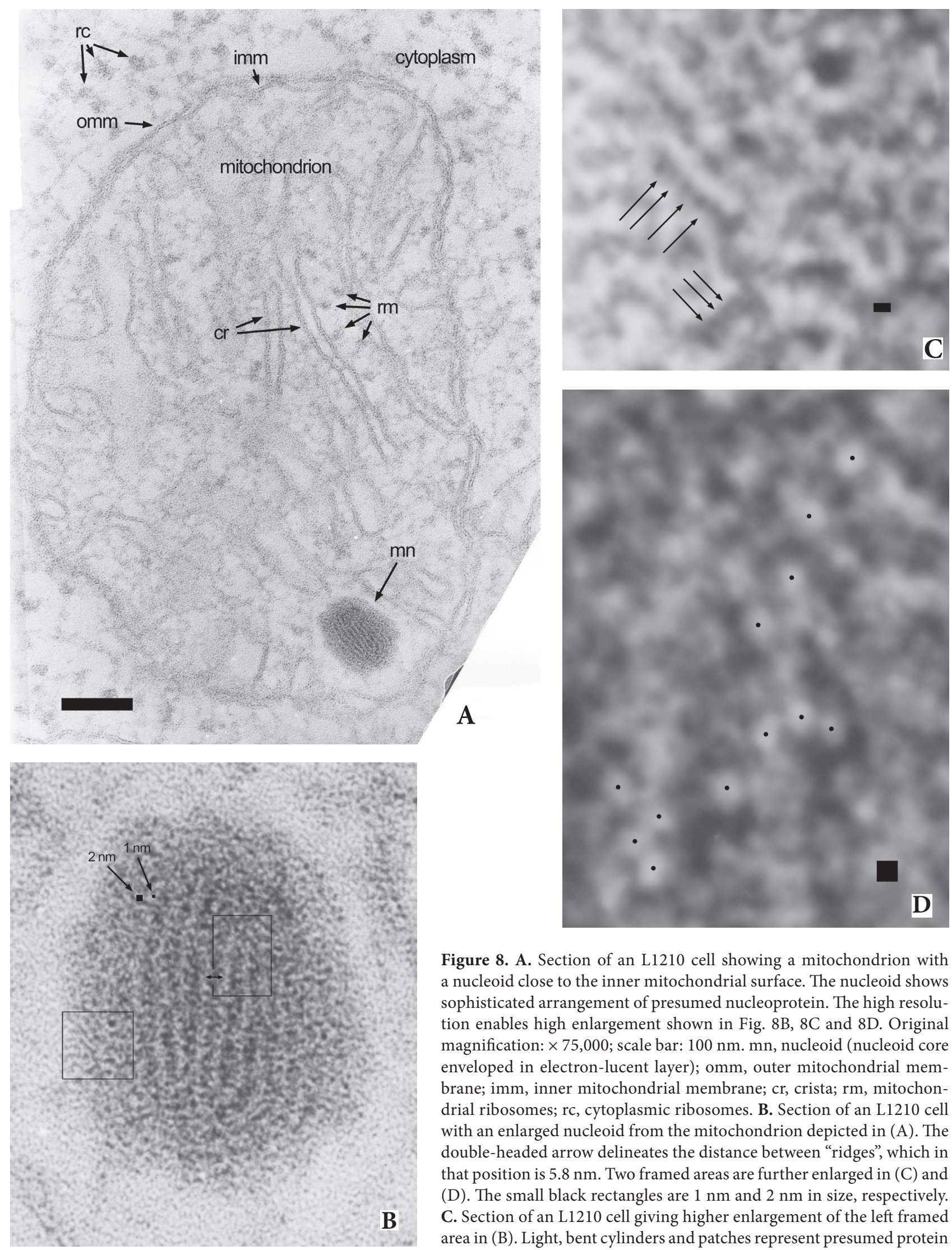

Figure 8. A. Section of an L1210 cell showing a mitochondrion with a nucleoid close to the inner mitochondrial surface. The nucleoid shows sophisticated arrangement of presumed nucleoprotein. The high resolution enables high enlargement shown in Fig. 8B, 8C and 8D. Original magnification: $\times 75,000$; scale bar: $100 \mathrm{~nm}$. $\mathrm{mn}$, nucleoid (nucleoid core enveloped in electron-lucent layer); omm, outer mitochondrial membrane; imm, inner mitochondrial membrane; cr, crista; rm, mitochondrial ribosomes; rc, cytoplasmic ribosomes. B. Section of an L1210 cell with an enlarged nucleoid from the mitochondrion depicted in (A). The double-headed arrow delineates the distance between "ridges", which in that position is $5.8 \mathrm{~nm}$. Two framed areas are further enlarged in (C) and (D). The small black rectangles are $1 \mathrm{~nm}$ and $2 \mathrm{~nm}$ in size, respectively. C. Section of an L1210 cell giving higher enlargement of the left framed area in (B). Light, bent cylinders and patches represent presumed protein 
the rows look like ridges, this is an optical illusion. Light, gray and dark spots are all at the same level because the section is planar. The nucleoid core edge is less electron-dense than the center and the chains of presumed proteins ranging in thickness from over $1 \mathrm{~nm}$ to less than $2 \mathrm{~nm}$ can be discerned. This is shown in greater detail in Figure 8C, which is an enlargement of the left framed area in Figure 8B. The surface of the section inside the nucleoid core shows many circular or elliptical spots with a diameter of over $1 \mathrm{~nm}$ (see Fig. 8D which represents the enlarged right framed area in Fig. 8B). We presume that these spots are protein chains sectioned perpendicularly to the chain axis or that the sections are skew. The occurrence of such sections assumes a much higher geometric probability than the presence of sections running parallel to the cylinder of the protein's alpha helix.

How to explain the regularity of the nucleoid nucleoprotein? Its substance lies in the regularity of the major component, DNA. The double helix is based on a carbohydratephosphate skeleton, which is fully regular. If this skeleton is saturated by mitochondrial transcription factor-A (TFAM) protein, which bends the double helix, the result is the thicker filament of the nucleoprotein. To arrange these filaments alongside each other, only weak (but many) bonds (for example hydrophobic and/or hydrogen ones) are required. For bending nucleoprotein filaments on the surface of the nucleoid core, some other components besides TFAM are probably needed.

\section{Resolution power}

To the best of our knowledge, we achieved an approximately three times higher resolution in cell section than has ever been published (Figs. 1A, 2, 4 and 8). One field of the nucleoid core edge shown in the left framed area of Figure $8 \mathrm{~B}$ was enlarged in Figure 8C. Arrows direct to the light faint connections between presumed protein chains. Their thickness is less than $0.5 \mathrm{~nm}$. These findings are detailed in the "Discussion" section.

chains with thickness ranging from more than $1 \mathrm{~nm}$ to less than $2 \mathrm{~nm}$. The arrows point to light faint connections between presumed protein chains. Their thickness is less than $0.5 \mathrm{~nm}$. These findings are discussed in detail in the "Discussion" section. Scale bar: $1 \mathrm{~nm}$. D. Section of an L1210 cell with higher enlargement of the right framed area in (B). This shows the nucleoid interior. The micrograph has many circular or elliptical light spots with diameter over $1 \mathrm{~nm}$ (some of these are marked by dots). We presume that these spots are protein chains sectioned perpendicular to the chain axis, or they may be skew sections. Small globular-like proteins may also be present. Black rectangle represents $1 \mathrm{~nm}$.

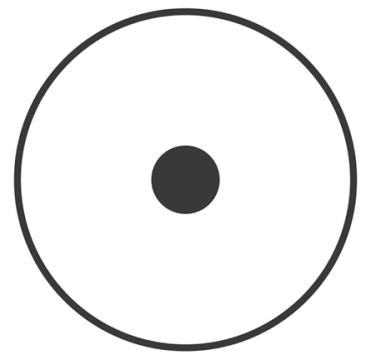

Figure 9. Comparison of relative cathode areas emitting electrons which pass through the specimens. Here the commonly used setting is compared with the newly established recording setting used in our laboratory. If the area of white plus black circle represents part of the area emitting electrons on the cathode, which is used for passing the specimen at the chosen exposure time of $3.6 \mathrm{~s}$, then the area of the black circle represents the area emitting electrons on the cathode used for passing the specimen when the exposure time is set to $90 \mathrm{~s}$. The white area depicting electrons further from the optical axis is lost or "filtered out" in the $90 \mathrm{~s}$ exposure by underfocused setting of the second condenser lens.

\section{Discussion}

\section{Records of nucleoid morphology in L1210 cells}

Based on morphological criteria, we divide L1210 cell nucleoids into the following 4 groups:

(1) The first group has sophisticated packaging of the most organized nucleoprotein. Juxtaposed filaments resemble fingerprint. The core is covered with light layer - envelope. Although this type is found very rarely, its frequency is highly underestimated due to technical reasons. It is difficult to imagine transcription occurring in such a complexly packed structure (Figs. 7 and 8 ).

(2) The second, most common L1210 cell nucleoid records show highly packed and very electron-dense circular or slightly oval-shaped cores with the same electron density as nucleoid cores of the first group but with no regular internal arrangement distinguishable. The cores are also covered with light envelope. Again, it is difficult to imagine that transcription occurs in such packed structures (Figs. 1A and 4). The apparent disorder inside the core may be a false finding due to the method used. Because the likelihood of sectioning parallel to the nucleoprotein filaments is extremely low, the situation recorded in Fig. 8A may be an exceptional coincidence. Consequently, the first two groups may in fact be identical, with regular internal organization, though the finding of regularity has a very low probability for technical reasons.

(3) The third group of nucleoids has anchorage to both inner and outer mitochondrial membranes. The part intervening the membranes is less electron-dense and then lighter than the nucleoid core but denser than proteins (Fig. 3; see also Prachar 2010). It is thus presumably a mixture of proteins and nucleic acids with higher protein content 
and this might represent an active replisome. If this holds, nucleoid replication occurs outside the nucleoid core. The replication machinery would be connected to the core but spatially would not be integral part of the core (Meeusen and Nunnari 2003).

Nucleoids of all three groups observed in L1210 cells are electron-dense, more packed than heterochromatin and they are mostly surrounded by a thick electron-lucent layer - envelope, presumably subdivided by walls into tens of compartments (chambers).

(4) Except these three groups of nucleoids, we observed only one nucleoid anchored into crista membranes (Fig. 2). Because it is one case only, we will not speculate about its purpose.

We would like to underline that difference in morphology always mirrors a difference in composition. Thus the isolated nucleoids (e.g. from HeLa cells) would be a mixture of the four described groups. We can assume that the protein composition of inactive and replicating nucleoids differs considerably. A protein composition mixture will be found in isolated nucleoids. This situation could be solved by comparison of highly active cells, e.g. in the early exponential phase, with dormant ones, e.g. serumstarved cells.

L1210 leukemia cells use anaerobic-like glycolysis as their major energy source (Charni et al. 2010). The rough endoplasmic reticulum is only exceptionally found close to mitochondria and the mitochondrial matrix is light-colored - electron-lucent. Interestingly, we found only packed nucleoids in this cell line both in interphase and mitosis (several hundred nucleoids inspected) (Figs. 1A, 4, 7 and 8). This could be explained by relatively weak activity and turnover as mitochondria are mostly not engaged in oxidative phosphorylation. This is the fundamental difference in Caco-2 cells, where many nucleoids are less electron-dense and very often even hardly discernible on the matrix background (Prachař 2010). On the basis of comparison with Caco-2 cells, we speculate that the L1210 electron-dense cores are inactive, awaiting eventual activation where the first phase will be unpacking or partial unpacking.

\section{Caco-2 cell nucleoids}

Caco- 2 cells grow slowly with high extracellular mucus production. These cells contain several large nucleoli, which is typical of cells highly engaged in proteosynthesis. These colon carcinoma cells maintain many markers of the colon epithelial cells including their differentiation ability. Caco-2 cell rough endoplasmic reticulum is often very close to mitochondria, and it frequently even envelops the mitochondria. On rough reticulum, the ribosomes are usually present on the side closer to the mitochondrion, while on the opposite side they are rare (Fig. 5). The mitochondrial matrix is granulous and shows much higher electron density than do L1210 cells. A granulous electron-dense matrix can indicate efficient energy production accompanied by the presence of negatively charged phosphate groups on matrix proteins, mainly enzymes.

We presume that the major source of energy essential for highly active proteosynthesis is oxidative phosphorylation, although Caco-2 cells may additionally use also glycolysis (Carlson-Newberry and Costello 1997). The nucleoid core electron density is always less distinctive here than in L1210 cells. It is difficult to distinguish the light layer surrounding the nucleoid core (Fig. 5). Some nucleoids are diffuse and some are even hardly discernible from the surrounding matrix (Prachař 2010). We speculate that these very "sparse" nucleoids are most active in transcription and that most DNA is engaged outside the core.

A layered nature was recently suggested for the nucleoid, considering the function of the supposed layers (Bogenhagen et al. 2008). We observed the nucleoid core and the surrounding layer as morphological entities and hence we speculate on the function of these structures, especially in comparison of two cell lines with very different proteosynthetic activity and different mode of energy metabolism.

\section{Walls in the layer surrounding the nucleoid core}

Functions of the walls in the light layer surrounding the nucleoid core may: (1) to provide a mechanical support to retain the nucleoid core in the center of the light body and isolate it from an unsuitable environment and preventing intimate contact with cristae membranes (this would act as a protective insulator of inactive nucleoids); (2) to create storage chambers for important regulatory or executive proteins for immediate recruitment to particular nucleoid compartments.

These functions are not mutually exclusive. The question arises whether a chamber with proteinous walls is able to maintain an individual environment inside. Eukaryotic cells developed compartmentalization mostly by using phospholipid membranes, which are very effective in maintaining the internal environment of organelles and of the entire cell. In some cases, proteinous walls separating different environments are also utilized where phospholipid membranes are unusable. One of the most sophisticated examples of this solution is the barrier created inside the nuclear pore complex (Prachař 2012).

\section{Probability of finding regular arrangement of the molecular complexes in the nucleoid}

The probability of finding a regular arrangement of nucleoid molecular complexes depends on sections cut parallel to 
them. The complexes are only several nanometers thick, so the likelihood is extremely low (Figs. 7 and 8A). We record a two-dimensional picture, but we have to consider that in three dimensions the section has to go plane-parallel to the plane of the chains. Therefore, the finding of one instance of regular arrangement in thirty inspected nucleoids is a large underestimation. It cannot even be excluded that all nucleoids in the case of L1210 cells, which use glycolysis as a major energy source (Charni et al. 2010), possess a regular arrangement of the nucleoprotein. The regular internal nucleoprotein arrangement in nucleoids would suggest that the packaging of mtDNA is not any exception and is an ordered, repetitive process, as seen when nuclear DNA is packaged by histones into nucleosomes or by protamines into toroids. The random TFAM binding to mtDNA was suggested to suffice for nucleoid packaging in vitro (Kukat et al. 2015). Nucleoids of the proper size were synthesized but they were irregular. Therefore, it seems that in vivo, the ordered process in space and in time would need to engage more components than TFAM only.

Our method is not able to solve whether all compacted nucleoids have regular internal structure. In contrast to L1210 cells, the nucleoids in Caco- 2 cells with highly active proteosynthesis are most probably not arranged in a similar manner because they are less electron-dense and hence more "sparse" (Fig. 5). The nucleoids in these cells are also smaller than those in L1210 cells and it is thus also possible that a certain portion of the DNA is engaged in transcription further from the nucleoid core and not only translation of m-RNA, as recently proposed (Bogenhagen 2012). Indeed, the nucleoid may be an even more dynamic structure owing to its functionality than we currently surmise.

\section{Frequency of finding nucleoids anchored in membranes}

The probability of finding nucleoids anchored in the membranes is low, not only due to technical reasons but also because their presence at that site may be indicative of the replicative stage (Meeusen and Nunnari 2003; Rajala et al. 2014). In this case, our model of L1210 cells is not appropriate to determine the incidence of anchored nucleoids, because our cells are at the end of the exponential phase and they do not need to propagate mitochondria and replicate nucleoids. Cells in the early exponential growth phase would be ideal in searching for replicating nucleoids.

\section{Speculation about the regulation of nucleoid activation}

In vitro experiments have shown that TFAM concentrations 2-3-fold higher than optimal stimulatory concentrations are sufficient to shut down transcription (Antoshechkin and Bogenhagen 1995). This is in agreement with the regulatory role of TFAM on the transcription level as TFAM is known to wrap, bend and in a dimer form to compact DNA (Diffley and Stillman 1992; Antoshechkin et al. 1997; Kukat and Larsson 2013; Ngo et al. 2014). Recently published work (Farge et al. 2014) shows that small variations in the TFAM-to-mtDNA ratio may be used to regulate mitochondrial gene transcription and DNA replication. A further finding supporting a regulatory role of TFAM in transcription is that higher numbers of TFAM molecules in mature stages of the Xenopus oocyte coincide with mitochondrial transcription down-regulation. The protein accumulated in mature oocytes may package mtDNA in complexes that prevent access to other regulatory proteins (Shen and Bogenhagen 2001). This is in agreement with our finding of heavily packed nucleoids in serum-starved cultured cells (Fig. 7, and Prachař 2010) and that of less packed nucleoids in Caco- 2 cells showing strong proteosynthesis. The TFAM levels bound in the nucleoid must be precisely regulated by incoming signals from the cytoplasm.

The nucleoid core - dark body, is situated inside the light body and the entire surface of the packed nucleoprotein is influenced by the content of the light body. This light zone may not only be protein in nature but may also contain small soluble components such as ions and water that can be substituted by epoxy resin during the embedding procedure. Unfortunately, the proportion of epoxy resin present cannot be currently determined by our high resolution method. Changes in the light body's environment could rapidly influence nucleoid composition. We speculate that a low-molecular-weight compound regulates TFAM nucleoid binding; and one such candidate with regulatory potential might be calcium cations (Jacob et al. 1994).

\section{Nucleoid size and mtDNA number per nucleoid}

The light layer surrounding the electron-dense core may cause the wide range in nucleoid size determined by different methods. In case that the particular light microscopy method used for nucleoid identification, including superresolution methods, is based on the detection of a particular protein present in the surrounding layer, the diameter of the electron-lucent body is recorded.

The 50 to $100 \mathrm{~nm}$ nucleoid core diameter in our preparations can be weighted by error to the lower value. Some of these smaller values may represent nucleoid spherical caps caused by off-center sectioning. Nevertheless, the 60 to 100 $\mathrm{nm}$ value consents with those obtained using antibodies directed to DNA (Kukat et al. 2011). Moreover, primary and secondary antibody dimensions increase the actual nucleoid core diameter. When we consider the number of genomes in one nucleoid, we have to remember that doubling the nucleoid core diameter gives an eight-fold increase in volume. It is reasonable to consider that the difference in nucleoid core 
volumes is not caused only by the protein content but also by the DNA content because electron density of nucleoid cores with different diameters shows the same appearance. We therefore hypothesize that nucleoids with $100 \mathrm{~nm}$ core diameter contain several $\mathrm{mtDNAs}$. This may be in agreement with the finding that certain portion of mtDNA is isolated in catenated form (Pohjoismäki and Goffart 2011). The size of nucleoids may depend also on culture conditions (Miyakawa et al. 2004) or on the mode of energy production by glycolysis versus oxidative phosphorylation.

\section{Sample preparation}

The use of very hard epoxy resin enabled very thin section preparation and drift elimination - prerequisites to achieve high resolution. Sections of a small area were placed on high number mesh grids, which is necessary in case of very thin sections, and moreover, it also eliminates drift. These conditions are based on accepted electron microscopic techniques.

\section{High resolution method of cell section visualization}

This study provides a direct extension of our earlier work (Prachař 2010). New findings in this work result from the new high resolution method recently developed in our laboratory and described and discussed in detail in a previously published work (Prachař 2014).

We used an enlarged field of the nucleoid edge to illustrate resolution power. Figure $8 \mathrm{C}$ is an enlargement of the left framed area in Figure 8B. Thin and faint connections between presumed protein chains demonstrate how high resolution can be achieved using our method. We investigated the nature of the thin filaments. They are very often present in our sections and they are too long and thin to be a protein side-chain. We presume that these thin filaments are artifacts generated during sample preparation. Nevertheless, these artifacts are suitable objects for testing the resolution power. It is interesting that the length of these thin filaments corresponds to the length of the glutaraldehyde molecule used in fixation. We used one particular place to highlight resolution where connections in two positions are roughly perpendicular to one another. We concluded that the presence of connections as such is not the effect of drift or astigmatism. Note: The technical resolution of our microscope is $1.4 \AA$ and therefore still several times higher than the resolution obtained in cell sections.

\section{Microscope settings}

Our high resolution method uses two microscope settings. The first is the commonly used setting for radiation-sensitive biological samples. We used sections of cells embedded in epoxy resin to search for objects of interest and to focus on them. The first condenser lens is set to spot size two $(2 \mu \mathrm{m}$ diameter). Searching is performed under lower magnification to avoid radiation damage. Then the desired higher magnification is set and focusing is performed outside the object. Only tuning of focusing is performed on the object. After focusing, the filament is turned off. We then change the setting of the first condenser lens to spot size number 5 (diameter $500 \mathrm{~nm}$ in our JEOL JEM-1200 EX microscope) and we set brightness to underfocused position (counterclockwise). When sections are very thin, we wait 15 minutes at this point to prevent specimen drift. Thereafter, the beam current is returned to the saturated position. There is no illumination on the screen. We then turn the brightness knob (the second condenser lens), clockwise towards the focus position. As soon as a weak illumination is seen, the exposure meter shows low current density. We set exposure to 90 seconds and took a photo. The image on the screen is so dark that it would not be possible to focus it. But the object is already focused. Most importantly, using this setting of both condenser lenses, we select electrons close to the optical axis. These possess not only a very similar direction but also practically the same energy. This selection has unforeseen effects on resolution. In addition, the long exposure time favors reversibility of some damaging reactions caused by the electron beam striking the specimen. The small diameter of the spot size, or the probe in general, is the strategy of "physical" electron microscopes with atomic resolution. The strategy used to obtain electrons with quasi-uniform energy is in that case energy-filtering.

Only practical application would prove whether our strategy is of advantage on using microscopes with stronger emitting guns like $\mathrm{LaB}_{6}$ or Schottky field emission gun. Stronger emitters would enable electron selection even closer to the optical axis. We compared the area of the surface of filament tip emitting electrons used for passing the sample at exposure time $3.6 \mathrm{~s}$ versus $90 \mathrm{~s}$. The area selected for the record of the sample was $25 \times$ smaller (or the diameter of the beam was $5 \times$ smaller) in the case of 90 s exposure compared to $3.6 \mathrm{~s}$ exposure time (Fig. 9). If we expect the same final intensity recorded on the film for both short- and long-term exposure, in case of $90 \mathrm{~s}$ exposure time, we use only $4 \%$ of emitted electrons per time unit compared with exposure time $3.6 \mathrm{~s}$. The emitted electrons used in long exposure are close to the optical axis and they are accelerated with a very similar force by the anode, therefore their energy (speed) is very similar (low energy spread) and accordingly, there is a minimal manifestation of chromatic aberration of the lenses. Spherical aberrations are also minimal because the electrons employed have periaxial, quasi-optimal trajectories - the beam is quasi coherent.

We routinely work at $75,000 \times$ magnification. We registered the first visible signs of radiation damage at 100,000x magnification in case of very thin sections. 
The radiation damage is roughly proportional to the total radiation dose. Resolution is roughly proportional to magnification up to a certain limit. However, a twice higher magnification requires a four times higher radiation dose to obtain a record signal of the same intensity. Thus to avoid radiation damage, it is more beneficial to use high resolution detectors than to use higher magnification.

It follows from our high resolution strategy that the microscope lenses reveal only low aberration effects. The cause of the low resolution often seen in biological preparations lies in improper use of electrons at the start of their trajectories, i.e. on the tip of the electron gun.

Improper selection of electrons just emitted from the gun is caused by inappropriate handling of the condenser lenses. Biologists commonly use electrons which are nonhomogenous in direction and energy to pass through the specimen. When this occurs, no other part of the optical system under the specimen can help to obtain high resolution.

Please note that in addition to the micrographs obtained by our recently introduced high resolution method, we also present four micrographs with usual resolution from our earlier series because of the very low frequency of situations recorded (Figs. 3, 5, 6 and 7).

\section{Concluding remarks}

This study presents new nucleoid morphological features obtained mainly by the high resolution strategy of transmission electron microscopy recently introduced in our laboratory. This strategy depends on appropriate sample preparation but above all on the new protocols of setting the microscope. The principle of the high resolution method is based on "feeding" the optical system with maximum homogenous electrons emitted from the center of the cathode. We refer to this method as "Energy filtering without filter". Great effort has been focused on avoiding radiation damage. This "long exposure method" can also be applied in other procedures such as immunoelectron microscopy and negative staining. It is very likely that even higher resolution can be achieved in case of radiation-sensitive biological samples by additional modifications of this method and also by changing some components of the microscope, such as apertures and electron gun.

Although the resulting high resolution image is occasionally "unreadable", past experience has shown that each method with improved resolution does invariably yield new knowledge.

Acknowledgements. We are grateful to Mrs. Margita Šulíková for providing cell cultures. This investigation was supported by the Slovak Cancer Research Foundation.

\section{References}

Antoshechkin I., Bogenhagen D. F. (1995): Distinct roles for two purified factors in transcription of Xenopus mitochondrial DNA. Mol. Cell. Biol. 15, 7032-7042 http://dx.doi.org/10.1128/MCB.15.12.7032

Antoshechkin I., Bogenhagen D. F., Mastrangelo I. A. (1997): The HMG-box mitochondrial transcription factor xl-mtTFA binds DNA as a tetramer to activate bidirectional transcription. EMBO J. 16, 3198-3206

http://dx.doi.org/10.1093/emboj/16.11.3198

Bogenhagen D. F., Rousseau D., Burke S. (2008): The layered structure of human mitochondrial DNAnucleoids. J. Biol. Chem. 283, 3665-36175

http://dx.doi.org/10.1074/jbc.M708444200

Bogenhagen D. F. (2012): Mitochondrial DNA nucleoid structure. BBA Gene. Regul. Mech. 1819, 914-920

doi: 10.1016/j.bbagrm.2011.11.005

Bogenhagen D. F., Martin D. W., Koller A. (2014): Initial steps in RNA processing and ribosome assembly occur at mitochondrial DNA nucleoids. Cell Metab. 19, 618-629 http://dx.doi.org/10.1016/j.cmet.2014.03.013

Brown T. A., Tkachuk A. N., Shtengel G., Kopek B. G., Bogenhagen D. F., Hess H. F., Clayton D. A. (2011): Superresolution fluorescence imaging of mitochondrial nucleoids reveals their spatial range, limits, and membrane interaction. Mol. Cell Biol. 31, 4994-5010 http://dx.doi.org/10.1128/MCB.05694-11

Carlson-Newberry S. J., Costello R. B. (1977): Assessment of cellular dysfunction during physiologic stress, emerging technologies for nutrition research: potential for assessing military performance capability. (Chapter 19). In: Assessment of Cellular Dysfunction During Physiologic Stress. pp. 401-414, Washington (DC): National Academies Press (US)

Charni S., Bettignies G., Rathore M. G., Aguiló J. I., van den Elsen P. J., Haouzi D., Hipskind R. A., Enriquez J. A., Sanchez-Beato M., Pardo J., Anel A., Villalba M. (2010): Oxidative phosphorylation induces de novo expression of the MHC class I in tumor cells through the ERK5 pathway. J. Immunol. 185, 3498-3503 http://dx.doi.org/10.4049/jimmunol.1001250

Diffley J. F., Stillman B. (1992): DNA binding properties of an HMG1-related protein from yeast mitochondria. J. Biol. Chem. 267, 3368-3374

Farge G., Mehmedovic M., Baclayon M., van den Wildenberg S. M. J. L., Roos W. H., Gustafsson C. M., Wuite G. J., Falkenberg M. (2014): In vitro-reconstituted nucleoids can block mitochondrial DNA replication and transcription. Cell Reports 8, 66-74 http://dx.doi.org/10.1016/j.celrep.2014.05.046

Hensen F., Cansiz S., Gerhold J. M., Spelbrink J. N. (2014): To be or not to be a nucleoid protein: A comparison of massspectrometry based approaches in the identification of potential mtDNAnucleoid associated proteins. Biochimie 100, 219-226 http://dx.doi.org/10.1016/j.biochi.2013.09.017

Holt J., He J., Mao C. C., Boyd-Kirkup J. D., Martinsson P., Sembongi H., Reyes A., Spelbrink J. N. (2007): Mammalian mitochondrial nucleoids: Organizing an independently minded genome. Mitochondrion 7, 311-321 
http://dx.doi.org/10.1016/j.mito.2007.06.004

Iborra F. J., Kimura H., Cook P. R. (2004): The functional organization of mitochondrial genomes in human cells. BMC Biol. 2,9 http://dx.doi.org/10.1186/1741-7007-2-9

Jacob W. A., Bakker A., Hertsens R. C., Biermans W. (1994): Mitochondrial matrix granules: their behavior during changing metabolic situations and their relationship to contact sites between inner and outer mitochondrial membranes. Microsc. Res. Techniq. 27, 307-318 http://dx.doi.org/10.1002/jemt.1070270406

Ježek P., Plecitá-Hlavatá L., Smolková K., Rossignol R. (2010): Distinctions and similarities of cell bioenergetics and the role of mitochondria in hypoxia, cancer, and embryonic development. Int. J. Biochem. Cell Biol. 42, 604-622 http://dx.doi.org/10.1016/j.biocel.2009.11.008

Kopek B. G., Shtengel G., Xu C. S., Clayton D. A., Hess H. F. (2012): Correlative 3D superresolution fluorescence and electron microscopy reveal the relationship of mitochondrial nucleoids to membranes. Proc. Natl. Acad. Sci. U.S.A. 109, 6136-6141 http://dx.doi.org/10.1073/pnas.1121558109

Kukat C., Wurm C. A., Spåhr H., Falkenberg M., Larsson N.-G., Jacobs S. (2011): Super-resolution microscopy reveals that mammalian mitochondrial nucleoids have a uniform size and frequently contain a single copy of mtDNA. Proc. Natl. Acad. Sci. U.S.A. 108, 13534-13539 http://dx.doi.org/10.1073/pnas.1109263108

Kukat C., Larsson N. G. (2013): mtDNA makes a U-turn for the mitochondrial nucleoid. Trends Cell Biol. 23, 457-463 http://dx.doi.org/10.1016/j.tcb.2013.04.009

Kukat C., Davies K. M., Wurm C. A., Spåhr H., Bonekamp N. A., Kühl I., Joos F., Polosa P. L., Park C. B., Posse V. et al. (2015): Cross-strand binding of TFAM to a single mtDNA molecule forms the mitochondrial nucleoid. Proc. Natl. Acad. Sci. U.S.A. 112, 11288-11293 http://dx.doi.org/10.1073/pnas.1512131112

Lee K. W., Okot-Kotber C., LaComb J. F., Bogenhagen D. F. (2013). Mitochondrial ribosomal RNA (rRNA) methyltransferase family members are positioned to modify nascent rRNA in foci near the mitochondrial DNA nucleoid. J. Biol. Chem. 288, 31386-31399 http://dx.doi.org/10.1074/jbc.m113.515692

Legros F., Malka F., Frachon P., Lombès A., Rojo M. (2004): Organization and dynamics of human mitochondrial DNA. J. Cell Sci. 117, 2653-2662 http://dx.doi.org/10.1242/jcs.01134

Meeusen S., Nunnari J. (2003): Evidence for a two membranespanning autonomous mitochondrial DNA replisome. J. Cell Biol. 163, 503-510 http://dx.doi.org/10.1083/jcb.200304040

Miyakawa I., Miyamoto M., Kuroiwa T., Sando N. (2004): DNA content of individual mitochondrial nucleoids varies depending on the culture conditions of the yeast Saccharomyces cerevisiae. Cytologia 69, 101-107 http://dx.doi.org/10.1508/cytologia.69.101
Ngo H. B., Lovely G. A., Phillips R., Chan D. C. (2014): Distinct structural features of TFAM drive mitochondrial DNA packaging versus transcriptional activation. Nat. Commun. 5, 3077 http://dx.doi.org/10.1038/ncomms4077

Pohjoismäki J. L., Goffart S. (2011): Of circles, forks and humanity: Topological organisation and replication of mammalian mitochondrial DNA. Bioessays 33, 290-299 http://dx.doi.org/10.1002/bies.201000137

Prachař J. (2010): Mouse and human mitochondrial nucleoid - detailed structure in relation to function. Gen. Physiol. Biophys. 29, 160-174 http://dx.doi.org/10.4149/gpb_2010_02_160

Prachař J. (2012): Structural features of transversal barrier in central channel of nuclear pore complex. Exp. Cell Res. 318, 614-622 http://dx.doi.org/10.1016/j.yexcr.2012.01.015

Prachař J. (2014): Spoke ring and anchorage of nuclear pore complex revealed by high resolution transmission electron microscopy. Gen. Physiol. Biophys. 33, 411-423 http://dx.doi.org/10.4149/gpb_2014013

Rajala N., Gerhold J. M., Martinsson P., Klymov A., Spelbrink J. N. (2014): Replication factors transiently associate with mtDNA at the mitochondrial inner membrane to facilitate replication. Nucleic Acids Res. 42, 952-967 http://dx.doi.org/10.1093/nar/gkt988

Seidel-Rogol B. L., Shadel G. S. (2002): Modulation of mitochondrial transcription in response to mtDNA depletion and repletion in HeLa cells. Nucleic Acids Res. 30, 1929-1934 http://dx.doi.org/10.1093/nar/30.9.1929

Shen E. L., Bogenhagen D. F. (2001): Developmentally-regulated packaging of mitochondrial DNA by the HMG-box protein mtTFA during Xenopus oogenesis. Nucleic Acids Res. 29, 2822-2828 http://dx.doi.org/10.1093/nar/29.13.2822

Spelbrink J. N. (2010): Functional organization of mammalian mitochondrial DNA in nucleoids: history, recent developments, and future challenges. IUBMB Life 62, 19-32 doi: 10.1002/iub.282

Tauber J., Dlasková A., Šantorová J., Smolková K., Alán L., Špaček T., Plecitá-Hlavatá L., Jabůrek M., Ježek P. (2013): Distribution of mitochondrial nucleoids upon mitochondrial network fragmentation and network reintegration in HEPG2 cells. Int. J. Biochem. Cell Biol. 45, 593-603 http://dx.doi.org/10.1016/j.biocel.2012.11.019

Wessels H. J., Vogel R. O., Lightowlers R. N., Spelbrink J. N., Rodenburg R. J., van den Heuvel L. P., van Gool A. J., Gloerich J., Smeitink J. A., Nijtmans L. G. (2013): Analysis of 953 human proteins from a mitochondrial HEK293 fraction by complexome profiling. PLoS One 8, e68340 http://dx.doi.org/10.1371/journal.pone.0068340

Received: December 20, 2015

Final version accepted: February 19, 2016

First published online: May 13, 2016 